Table 1. Investigation outcome summary

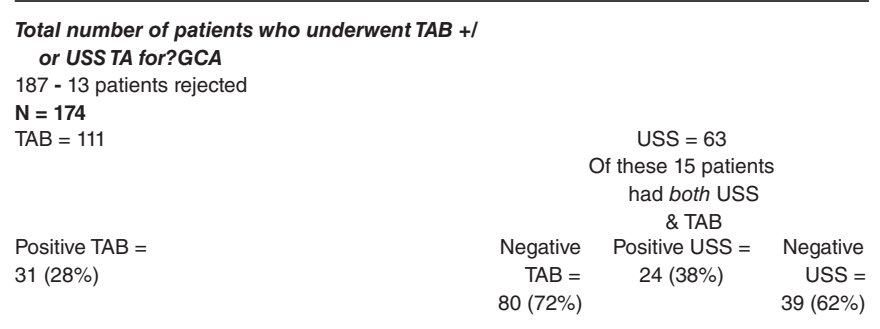

Conclusion: Our study, highlights that a probability score for GCA derived from a large multi-centre cohort of patients who were biopsy positive, predicts ultrasound positivity with similar accuracy. Our work reveals that scoring systems are not infallible but can be helpful in guiding clinical decision making

References:

[1] Ing EB, Lahaie Luna G, Toren A, et al. Multivariable prediction model for suspected giant cell arteritis: development and validation. Clin Ophthalmol. 2017;11:2031-2042. Published 2017 Nov 22.

Acknowledgments: Many thanks to the Rheumatology, Opthalmology \& Ultrasound teams at Royal Wolverhampton NHS Trust

Disclosure of Interests: None declared

DOI: 10.1136/annrheumdis-2020-eular.6597

\title{
AB0489 \\ BETA 2 MICROGLOBULIN AS A PROGNOSTIC FACTOR IN CRYOGLOBULINEMIA NON ASSOCIATED WITH HEPATOTROPIC VIRUSES
}

S. Jeria ${ }^{1,2}$, T. Franco ${ }^{3,4}$, A. Baucells $s^{3,4}$, A. García-Guillén ${ }^{2,5}$, D. Lobo Prat ${ }^{2,5}$, L. Sainz Comas ${ }^{2,5}$, H. Park ${ }^{2,5}$, A. M. Millán Arciniegas ${ }^{2,5}$, P. Moya ${ }^{2,5}$, A. Mariscal ${ }^{3,4}$, L. Alserawan ${ }^{3,4}$, A. Laiz ${ }^{2,5}$, B. Magallares ${ }^{1,2}$, C. Pitarch ${ }^{1,2}$, M. Riera ${ }^{1,2}$, C. Juarez ${ }^{3,4}, \mathrm{H}$. Corominas ${ }^{2,5}$. ${ }^{1}$ Hospital Universitari de la Santa Creu i Sant Pau \& Dos de Maig, Barcelona, Spain; ${ }^{2}$ Rheumatology Unit, Barcelona, Spain; ${ }^{3}$ Hospital Universitari de la Santa Creu i Sant Pau, Barcelona, Spain; ${ }^{4}$ Immunology Unit, Barcelona, Spain; ${ }^{5}$ Hospital Universitari de la Santa Creu i Sant Pau \& Dos de Maig, Barcelona, Spain

Background: Cryoglobulinemia (CG) is a rare phenomenon related to haematological disorders, infections and autoimmune diseases. Age and renal involvement are known prognostic markers.

Objectives: To describe the differential clinical features and the prognostic factors in a cohort of patients diagnosed with CG non-associated with hepatotropic viruses.

Methods: A descriptive, retrospective study of a cohort comprised of 252 cryoglobulin positive samples, obtained from the immunology laboratory database of a tertiary hospital attending 450,000 people over 1 year. 186 patients with CG positive samples were included, 87 of which were not associated with neither hepatitis B nor C virus. Demographic, clinical, serological and

Table 1. Clinical, epidemiological and serical characteristics of patients with CG

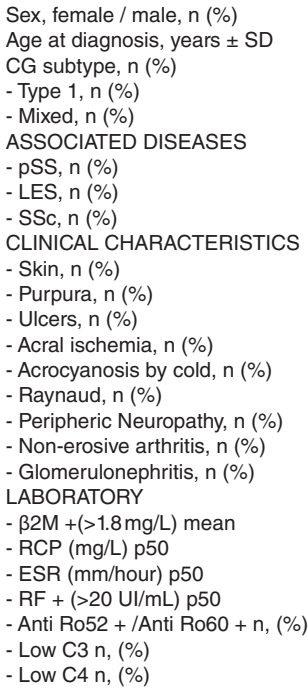

$65 / 22(74.7 / 25.3)$ $60 \pm 16$

$27(30)$

$61(70)$

$37(42,5)$

$9(10,3)$

$7(8,05)$

$30(34,5)$

14(16)

$5(5,7)$

$2(2,3)$

$7(8)$

$19(21,8)$

$17(19,5)$

$29(33,3)$

$10(11,5)$

3.9
3.7
28
124
$42(48.3)$
$48(55.1)$
$36(41.4)$

pathological data were collected. Nonparametric variables were compared using a Wilcoxon test.

Results: Out of 186 reviewed patients, 87 (46.7\%) are included in this study. The mean age at $C G$ diagnosis was $60( \pm 16)$ years. Mixed CG was the predominant subtype, detected in $66(75.9 \%)$ patients, 10 of which $(11.5 \%)$ were associated with glomerulonephritis (GN) with compatible biopsy, 17 (19.5\%) with peripheral neuropathy (PN), 29 (33.3\%) with non-erosive arthritis and 10 (11.5\%) with leukocytoclastic vasculitis confirmed by skin biopsy. The clinical, epidemiological and serological characteristics of the sample are summarized in Table 1.

In the comparative analysis of patients with $C G$ and Beta 2 microglobulin $(\beta 2 \mathrm{M}), \mathrm{CG}$ and rheumatoid factor $(\mathrm{RF})$, those with high $\beta 2 \mathrm{M}(>1.8 \mathrm{mg} / \mathrm{L})$ presented significantly more GN (p0.016) and PN (p0.013). However, the association of RF with either GN (p0.948) or PN (p0.645) was not significant. Also, high $\beta 2 \mathrm{M}$ was significantly related to complement consumption of $\mathrm{C} 4$ (p: 0.015) but not of C3 (p: 0.063). In the 30 (34.5\%) patients with skin manifestations, high $\beta 2 \mathrm{M}$ showed no statistically significant association. The main systemic autoimmune diseases associated were primary Sjögren's Syndrome (pSS) 37 (42.5\%), Systemic Lupus Erythematosus (SLE) 9 (10.3\%) and Systemic Sclerosis (SSc) 7 (8.05\%).

Conclusion: A direct association between presence of elevated levels of $\beta 2 \mathrm{M}$ and the existence of progression to glomerulonephritis and peripheral neuropathy is found in our cohort. No correlation is found between the presence of $C G$ and other serological markers of autoimmunity except low C4 $C G$ with elevated $\beta 2 \mathrm{M}$ does not associate with greater skin involvement or arthritis.

References:

[1] A.C. Desbois et al. Cryoglobulinemia: An update in 2019. Joint Bone Spine (2019)

[2] Cacoub P, Cryoglobulinemia Vasculitis, The American Journal of Medicine (2015)

Disclosure of Interests: None declared

DOI: 10.1136/annrheumdis-2020-eular.6229

\section{$\mathrm{AB} 0490$}

\section{CIRCULATING REGULATORY T CELLS WERE ABSOLUTELY DECREASED IN TAKAYASU'S ARTERITIS PATIENTS}

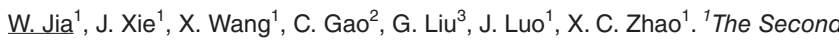
Hospital of Shanxi Medical University, Taiyuan, China; ${ }^{2}$ Brigham and Women's Hospital, Harvard Medical School, Boston, United States of America; ${ }^{3}$ The Second Clinical Hospital of Shanxi Medical University, Taiyuan, China

Background: Takayasu arteritis (TA) refers to chronic progressive non-specific inflammation that involves the aorta and its main branches, causing stenosis and occlusion of arteries in different parts, and ischemic manifestations in the corresponding parts. $A$ variety of immune dysfunctions are involved in the occurrence and development of $\mathrm{TA}^{(1)}$ Recent studies have shown that Th17/Treg imbalance plays an important role in the pathogenesis of Takayasu's arteritis, in which T help 17 cells (Th17) cells are up-regulated in TA patients ${ }^{(2)}$. Th17 cells are closely related to Treg cells during differentiation. There are few studies on the expression level of CD4+CD25+FOX3+T lymphocyte (Treg) cells. This study aims to study the clinical significance of Treg cell expression in peripheral blood of patients with Takayasu's arteritis.

Objectives: To analyze the levels of circulating lymphocyte subsets and serum cytokines in patients with takayasu arteritis (TA), and explore the relationship between their changes and TA disease activity.

Methods: A total of 46 TA patients and 43 gender-age-matched healthy controls were enrolled. According to the NIH standard, 30 patients were in active disease Flow cytometry was used to detect the absolute numbers and ratios of Th1, Th2, Th17 and Treg cells in peripheral blood of all subjects. Magnetic bead-based multiplex immunoassay was used to detect cytokines and statistical analysis was performed.

Results: Compared with the healthy controls, the absolute number and proportion of peripheral Treg cells of TA patients significantly decreased while those of Th17 cells increased significantly, leading to the increased ratio of Th17 / Treg Compared with the inactive group, the TA active group had significantly increased IL-6 and TNF-a, and there was no significant difference in the expression of Th17 cells and Treg cells.

Conclusion: In peripheral blood of TA patients, Treg cells decreased, while Th17 cells increased as compared with healthay controls, leading to an imbalance between Th17 and Treg cells. The levels of IL- 6 and TNF-a were related to disease activity.

References:

[1] Russo, R.A.G. and M.M. Katsicas, Takayasu Arteritis. Front Pediatr, 2018. 6: p. 265. 

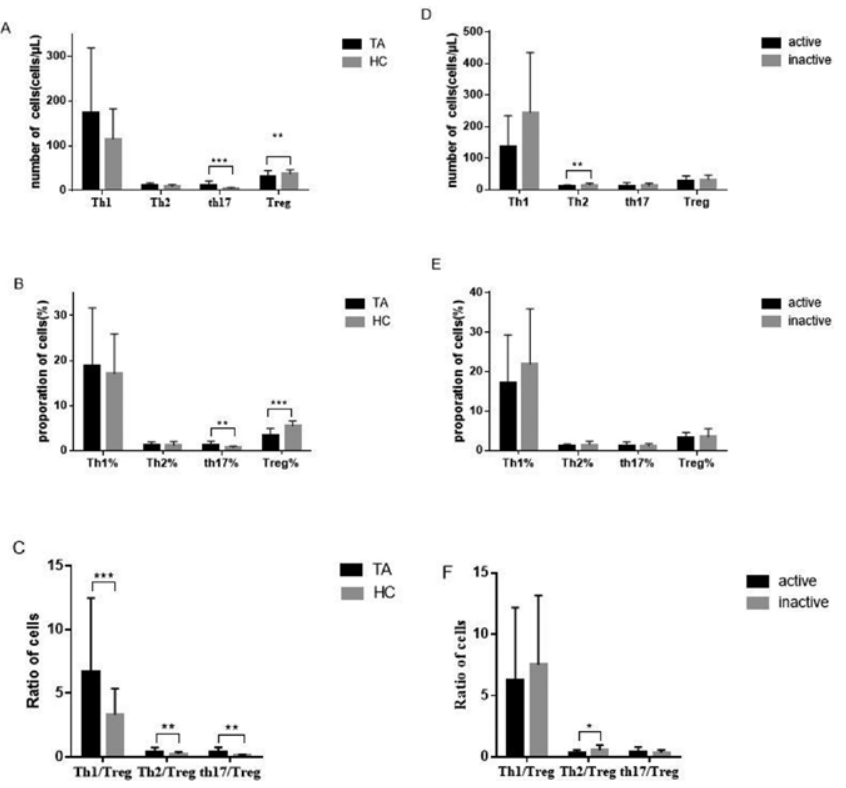

Figure 1. Characteristics of the absolute numbers and proportions of Th1cells, Th2cells, Th17 cells and CD4Treg cells in the PB of patients with TA.(A-C)The levels of Th17 cells and the ratio of Th1/Treg,Th2/Treg,Th17/Treg in PB were significantly increased in patients with TA $(n=46)$. The absolute number and the proportion of CD4Treg cells were significantly decreased in $T A(n=46)$. (D-F) The absolute number of Th2 cells and ratio of Th2/Treg in PB were significantly decreased in active patients with TA $(n=30)$. Neither the absolute number nor proporation of Th1, Th17 and Treg cells was altered significantly between active TA patients $(n=30)$ and inactive TA patients $(n=16) .{ }^{*} P<0.05 ;{ }^{*} \mathrm{*} P<0.001 . P<0.05$ was considered statistically significant. TA,takayasu arteritis; $\mathrm{PB}$ peripheral blood;Tregs, regulatory Tcells.

[2] Misra, D.P., S. Chaurasia, and R. Misra. Increased Circulating Th17 Cells, Serum IL-17A, and IL-23 in Takayasu Arteritis. Autoimmune Dis, 2016. 2016: p. 7841718 .

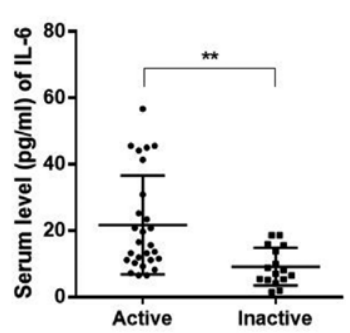

B

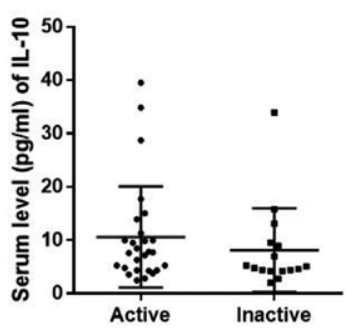

C

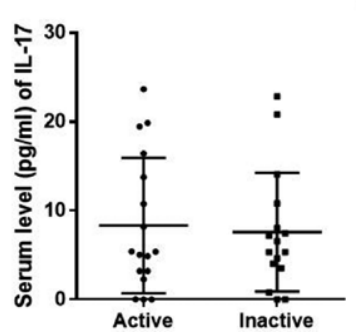

D

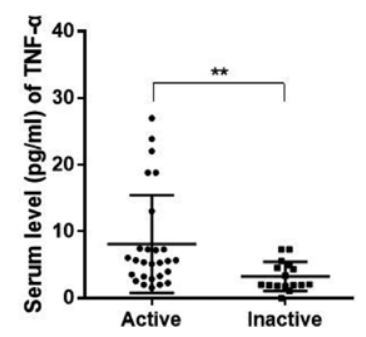

Figure 2. Characteristics of serum concentrations of cytokine (including IL-6, IL-10, IL-17 and TNFa) between active TA patients $(n=30)$ and inactive TA patients $(n=16) .(A, D)$ In terms of cytokines, the concentration of IL-6 and TNF-a was significantly up-regulated,(B,C)but no significant changes in IL-10, and IL-17 were found. ${ }^{*} P<0.05$; ${ }^{*} P<0.001$. $P<0.05$ was considered statistically significant.

Disclosure of Interests: None declared

DOI: 10.1136/annrheumdis-2020-eular.1068

\section{AB0491}

ELEVATED COMPLEMENT 3 INDICATES DISEASE ACTIVITY IN TAKAYASU ARTERITIS

R. Chen ${ }^{1}$, L. MA2, S. Wu ${ }^{1}$, L. MA2, L. Jiang ${ }^{1}$ on behalf of the East China Collaborative Group of Vasculitis. ${ }^{1}$ Zhongshan Hospital Fudan University,
Rheumatology, Shanghai, China; ' Zhongshan Hospital Fudan University, Rheumatology, Shanghai, China

Background: The disease activity evaluation of Takayasu arteritis (TA) is a critical issue for disease monitoring and treatment. But the previous markers such as Kerr score or ITAS 2010 are not convenient enough.

Objectives: We aim to explore novel biomarkers to assess TA disease activity. Methods: This cross-sectional study was based on the East China TA (ECTA) cohort. Demographic characteristics, clinical features, laboratory and imaging results were collected. Complements and their combination with other biomarkers in identifying active disease (Kerr >=2) group were analyzed. Internal and external validation were employed to confirm the accuracy and stability of the results.

Results: 519 patients were enrolled, among which 406 cases (72.2\%) were identified as active disease. Higher ESR, CRP, platelet, globulin, IgG, IL-6, complement 3 (C3), complement 4 (C4) and median haemolytic complement (CH50) levels were observed in the active disease group. Logistic regression analysis demonstrated that $\mathrm{C} 3$ levels [odds ratio [OR] $(95 \% \mathrm{Cl})$ : $10.710(1.825-62.835)$ $\mathrm{P}=0.009$ ] and CRP [OR $(95 \% \mathrm{Cl}): 1.041(1.009-1.073), \mathrm{P}=0.011]$ were independently associated with active disease. The cut-off of $\mathrm{C} 3$ to identify active TA was $1.085 \mathrm{~g} / \mathrm{L}$, with $69.9 \%$ sensitivity, $66.7 \%$ specificity. Combining the CRP (cutoff, $10.65 \mathrm{~g} / \mathrm{L}$; sensitivity, $50.7 \%$; specificity, $82.4 \%$ ) and C3, the sensitivity and specificity to identify the active disease were $85.1 \%$ and $55.0 \%$ (parallel test), and $35.4 \%$ and $94.1 \%$ (serial test), respectively. C3 could significantly improve the diagnostic ability of CRP [net reclassification index: OR $(95 \% \mathrm{Cl}): 1.728(1.556-$ 1.990), $\mathrm{P}=0.000$; integrated discrimination index: OR $(95 \% \mathrm{Cl}): 0.328(0.224$ $0.431), P=0.000]$. The accuracy of the 10 -fold cross validation of combining CRP with C3 was over $75 \%$, and the accuracy of the external validation with 53 TA cases was $72.73 \%$

Conclusion: $\mathrm{C} 3$ could reflect the disease activity of TA, and combining CRP with C3 could significantly improve the disease activity evaluation in TA.

\section{corences:}

[1] Ma J, Luo X, Wu Q, Chen Z, Kou L, Wang H. Circulation levels of acute phase proteins in patients with Takayasu arteritis. J Vasc Surg. 2010;51(3):700-6. Acknowledgments: This work was supported by the National Natural Science Foundation of China [NSFC 81771730 and 81601398].

Disclosure of Interests: : None declared

DOI: 10.1136/annrheumdis-2020-eular.2591

\begin{tabular}{|l|l}
\hline AB0492 & INTESTINAL MICROBIOTA COMPOSITION OF \\
& PATIENT'S WITH BEHCET'S DISEASE: DIFFERENCES \\
& BETWEEN EYE, MUCOCUTANEOUS AND VASCULAR \\
& INVOLVEMENT (RHEUMA-BIOTA STUDY)
\end{tabular}

N. S. Yasar Bilge ${ }^{1}$, V. Perez Brocal ${ }^{2}, \underline{\text { T. Kașifoğlu }}^{3}$, U. Bilge ${ }^{4}$, N. Kasifoglu ${ }^{5}$, A. Moya ${ }^{2,6,7}$, E. C. Dinleyici ${ }^{8} .{ }^{1}$ Eskisehir Osmangazi University, Medical Faculty, Department of Internal Medicine, Division of Rheumatology, Eskişehir, Turkey; ${ }^{2}$ Área de Genómica y Salud, Fundación para el Fomento de la Investigación Sanitaria y Biomédica de la Comunidad Valenciana (FISABIOSalud Pública), Valencia, Spain; ${ }^{3}$ Eskisehir Osmangazi University, Medical Faculty, Eskişehir, Turkey; ${ }^{4}$ Eskisehir Osmangazi University, Medical Faculty, Department of Family Medicine, Eskişehir, Turkey; ${ }^{5}$ Eskisehir Osmangazi University, Medical Faculty, Department of Microbiology, Eskişehir, Turkey; ${ }^{6}$ Institute for Integrative Systems Biology, Universitat de València, Valencia, Spain; ${ }^{7}$ CIBER en Epidemiología y Salud Pública (CIBEResp), Madrid, Spain; ${ }^{8}$ Eskisehir Osmangazi University, Medical Faculty, Department of Pediatrics, Eskişehir, Turkey

Background: Recently, it has been shown that changes in microbiota composition play a role in the etiology and pathogenesis of chronic diseases. Changes in oral and intestinal microbiota diversity and composition are suggested in Behcet disease (BD), however there are no study available about the potential gut microbiota changes among different clinical forms of BD.

Objectives: The aim of this study was to evaluate the intestinal microbiota composition of patient with BD and healthy controls, and also compare BD patients regarding to their eye, mucocutaneous and vascular involvement.

Methods: In this prospective cohort study,27 patients diagnosed with BD and 10 aged and sex matched healthy controls were included. Patients with a body mass index $>35$, who have used antibiotics or probiotics in the last 4 weeks, patients with chronic gastrointestinal or other systemic diseases, and those with acute / severe gastrointestinal symptoms requiring medical treatment were excluded from the study. For the intestinal microbiota analysis, gene amplification, library formation, sequence analysis and bioinformatic evaluation of the results were performed with 16SrRNA next generation sequencing methods with Illumina MiSeq.

Results: There was no difference between the BD group and the control group in terms of alpha (Chao-1 and Shannon) and beta (Bray-Curtis) microbiota diversity indices ( $p>0.05$ ). Actinomyces, Libanicoccus, Collinsella, Eggerthella, Enetrohabdus, Catenibacterium and Enterobacter were significantly higher in BD group compared to the 